\title{
Dissipative Quantum Computing with Majorana Fermions
}

\author{
Henning Soller \\ Institut für Theoretische Physik, Ruprecht-Karls-Universität Heidelberg, Heidelberg, Germany \\ Email: hsoller@googlemail.com
}

Received 28 December 2015; accepted 19 February 2016; published 22 February 2016

Copyright (C) 2016 by authors and Scientific Research Publishing Inc.

This work is licensed under the Creative Commons Attribution International License (CC BY). http://creativecommons.org/licenses/by/4.0/

c) (i) Open Access

\begin{abstract}
We describe a scheme for universal quantum computation with Majorana fermions. We investigate two possible dissipative couplings of Majorana fermions to external systems, including metallic leads and local phonons. While the dissipation when coupling to metallic leads to uninteresting states for the Majorana fermions, we show that coupling the Majorana fermions to local phonons allows to generate arbitrary dissipations and therefore universal quantum operations on a single $Q u B i t$ that can be enhanced by additional two-QuBit operations.
\end{abstract}

\section{Keywords}

\section{Dissipative Quantum Computing, Phonon Interaction, Majorana Fermions, Universal Quantum} Computation

\section{Introduction}

Majorana fermions in superconducting heterostructures provide one of the most challenging but also interesting candidates for quantum computation [1]-[4]. In a one dimensional topological superconductor, a single delocalized pair of Majorana bound states (MBSs) exists [5], one on the left $\left(\gamma_{L}\right)$ and one on the right $\left(\gamma_{R}\right)$. Interestingly, exchanging these positions leads to a topologically protected accumulated phase acquired by the MBSs [2] which makes them a possible starting point for topological quantum computation [6].

However, the above statement is only true as long as no coupling to an ungapped system is present [7]. If we do couple one of the MBSs, e.g. a metallic lead its state decoheres just as an ordinary fermionic subgap bound state [8].

In this paper, we want to demonstrate that the above feature of a MBS does not always hinder its applicability as a QuBit as it is previously assumed [8] but provides a new way of quantum computation on MBSs via dissipation [9]-[11]. We will approach dissipative quantum computing on MBSs in two steps: first, we consider 
a simple tunnel junction and show that not every state is subjected to dissipation in order to illustrate the basis of dissipative quantum computing. Second, we will consider a more involved setup to demonstrate the possibility of universal quantum computation.

\section{Tunnel Junction}

We start by considering two MBSs coupled to a metallic lead as shown in Figure 1.

The tunnel coupling can be described by the Hamiltonian

$$
H_{\text {tun }}=\lambda\left(\Psi^{+}-\Psi\right) \gamma_{R}+i \lambda\left(\Psi^{+}+\Psi\right) \gamma_{L}=\lambda \Psi^{+}\left(\gamma_{R}+i \gamma_{L}\right)-\lambda \Psi\left(\gamma_{R}-i \gamma_{L}\right)=\lambda \Psi^{+} \tilde{\gamma}-\lambda \Psi \tilde{\gamma}^{+} .
$$

$\gamma_{L}$ and $\gamma_{R}$ operate on the state of the Majorana fermion, which can either have even or odd parity and can be expressed using the fermion operators of the lead $\Psi$. Denoting their state by a four vector $\left(\right.$ even $_{R}$, odd $_{R}$, even $\left._{L}, \operatorname{odd}_{L}\right)$ the operators $\gamma_{L}$ and $\gamma_{R}$ can be written as Pauli spin matrices $\sigma_{x}$ and $\tilde{\sigma}_{x}$ operating on either the first or the last two columns. The operator $\tilde{\gamma}=\gamma_{R}-i \gamma_{L}$.

For a high bias voltage $V$ and low temperature we may assume the transitions from the MBSs to the lead to be irreversible. In this case the quantum mechanical tunneling operations can be reduced to simple quantum jumps [12] as given a certain direction for the bias voltage the reversed transport can also quantum mechanically be neglected. Experimentally this situation can be realized using the typical temperatures far below the gap of the material in which the Majorana fermions reside and a bias voltage far above this gap. Consequently the $\tilde{\gamma}$ 's can then be transformed to quantum jump operators which allows to write the time-evolution by a master equation in Lindblad form [12]

$$
\dot{\rho}=\mathcal{L}(\rho)=\sqrt{\Gamma}\left(2 \tilde{\gamma} \rho \tilde{\gamma}^{+}-\tilde{\gamma}^{+} \tilde{\gamma} \rho-\rho \tilde{\gamma}^{+} \tilde{\gamma}\right)
$$

where $\tilde{\gamma}=\left(\sigma_{x}+i \tilde{\sigma}_{x}\right)$ and $\Gamma=\pi \rho_{0} \lambda^{2}$ with the density of states in the metal $\rho_{0}$.

The Lindblad operator does not contain more involved tensor products of operators since we only consider simple tunneling processes on the left and right side so far [13].

Equation (4) has eigensolutions $\mathcal{L}\left(\rho^{*}\right)=0$ which do not decohere. Indeed, solving Equation (4) leads to

$$
\rho^{*}=\left(\begin{array}{cccc}
r_{11} & r_{12} & 0 & 0 \\
r_{12} & r_{22} & 0 & 0 \\
0 & 0 & r_{33} & r_{34} \\
0 & 0 & r_{34} & r_{44}
\end{array}\right),
$$

with $r_{i j}$ arbitrary numbers and $\rho^{*}$ properly normalised.

The first observation from this simple calculation is that dissipation can be engineered and certain states survive the dissipative evolution. However, in this simple case the states that survive are not very interesting for quantum computation since the off-diagonal elements which would give rise to entangled states between $\gamma_{L}$ and $\gamma_{R}$ vanish.

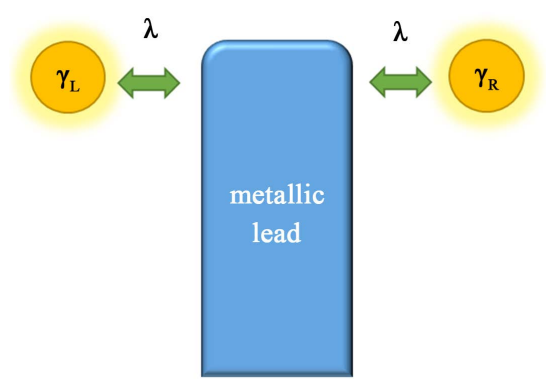

Figure 1. Two Majorana fermions on the left $\left(\gamma_{L}\right)$ and the right $\left(\gamma_{R}\right)$ are tunnel coupled to a metallic lead. 


\section{Phonon Coupling}

Therefore, we now discuss coupling of the MBSs to a phonon mode of an auxiliary contact. A phonon is a typical cause for dissipation and here we want to illustrate that it is also an interesting one. Coupling a phonon mode to a Majorana fermion $\gamma$ and an auxiliary lead (in $y$-direction) has the form [14]

$$
H_{\mathrm{tun}, x}=i t_{x} \hat{x}\left[\Psi^{+}(y=0)-\Psi(y=0)\right] \gamma,
$$

with the displacement operator $\hat{x}$ that has to be expressed by the bosonic operators corresponding to the phonon and the lead described by fermionic operators $\Psi$.

We should add that higher order tunneling terms may occur corresponding to terms $H_{\mathrm{tun}, x, n}=i t_{x, n} \hat{x}^{n}$, which correspond to phonon processes of higher order. In accordance with previous treatments of similar problems we only consider the lowest order as in Equation (6) in order to simplify the description [15]. Now, we do not want to couple the Majorana fermions to an outer lead [14] but rather couple them laterally as illustrated in Figure 2.

In this case the $x$-dependent coupling has the form

$$
H_{\mathrm{tun}, x}=\sum_{n, m=1}^{N} t_{x, n, m} \hat{x}_{n, m} \gamma_{n} \gamma_{m}
$$

where $\hat{x}=\left(b_{n m}^{+}+b_{n m}\right)$ is written in terms of bosonic phonon operators that correspond to the distance $x_{n}-x_{m}$.

In the language of dissipative quantum computing each phonon mode between two Majorana fermions provides an ancilla (by definition) the Majoranas are coupled to. The phonons should be coupled to an external bath such that they are described by a Lindblad equation with a Lindblad operator $L_{x, n, m}=\sqrt{t_{x, n, m}} \sigma_{-}$, where $\sigma_{-}=|0\rangle\langle 1|$ is the jump operator for a deexcitation of a phonon.

If the system is gapped (as it has to be for a superconducting system) the ordinary tunneling Hamiltonian discussed in Section 2 is not present and Equation (7) is the only remaining coupling of the Majoranas (the decoupled lead Hamiltonian of the superconductor can be disregarded).

As was shown in other works [6] one can associate Pauli matrices to different combinations of the Majorana operators. E.g. let us assume we have a single logical QuBit consisting of four Majorana fermions [16], then the two states of the logical QuBit are encoded as $|00\rangle$ and $|11\rangle$, corresponding to no excitations on both Majorana or both Majoranas being excited. These two states have the same (even) quasiparticle parity such that coherent superpositions are allowed. An arbitrary state has the form

$$
|\Psi\rangle=\alpha|00\rangle+\beta|11\rangle,|\alpha|^{2}+|\beta|^{2}=1,
$$

and the Pauli matrices are bilinear combinations of the four Majorana operators

$$
\sigma_{x}=-i \gamma_{2} \gamma_{3}, \sigma_{y}=i \gamma_{1} \gamma_{3}, \sigma_{z}=-i \gamma_{1} \gamma_{2} .
$$

All of these can be realized by couplings as in Equation (7) so that any Liouville operator can be constructed [11]. Mind, that this ensures completeness only of the one-QuBit operations since we have restricted ourselves to the states $|00\rangle$ and $|11\rangle$.

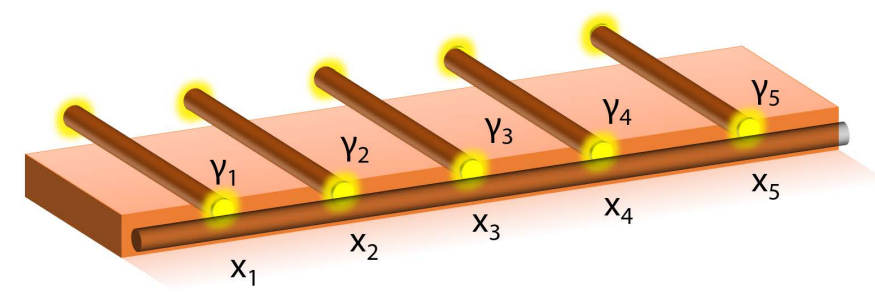

Figure 2. Five Majorana fermions are present in strongly spin orbit coupled quantum wires on top of a superconductor. These Majorana fermions are separated in $x$-direction and are all coupled to another lead e.g. a carbon nanotube (black). The carbon nanotube is gapped due to the proximity effect from the nearby superconductor but the Majorana fermions are all coupled to local phonon modes of the carbon nanotube. The first four Majorana fermions form a QuBit and the fifth one illustrates the possibility to add more QuBits in the same structure. 
This means that if the couplings $t_{x, n, m}$ are suitably chosen any state of the chosen Majorana QuBit can be produced. In order to allow for universal quantum computation for we also need specific operations that work on more than one Majorana QuBit. This other gate we need is not easily produced by a decoherence process since the Majorana QuBit we just discussed is said to be topologically protected from decoherence by the environment, meaning that bit-flip or phase-shift errors necessarily involve the change of pairs of Majorana QuBits or combinations of four Majorana operators (see Equation (9)).

The additional gate we need for universal quantum computation is the non-destructive measurement of the quasiparticle parity of any four MBSs [17]. In the case of Majorana particles realized in superconducting heterostructures this is most easily realized using a Josephson junction for charge read-out [7]. A non-demolition measurement can be realized using a similar setup [18] to the one shown in Figure 2 with the addition of a Josephson junction with a well-defined phase difference $\Phi$ (see Figure 3).

In this case, we would realize two Majorana QuBits using eight Majorana fermions, again employing strongly spin-orbit coupled semiconductor nanowires with the additional possibility of tuning the position of the Majorana fermions in these wires by side gates. The read-out of the phase difference amounts to a measurement of the quasiparticle parity of the state and therefore the desired gate operation [18].

Since the dissipative part of this setup is the new feature, in the remainder of this paper we will describe in more detail how to obtain the desired states in the single Majorana QuBit by dissipative quantum computing.

\section{Realizing Specific States in the Majorana QuBit}

Let us study the actual evolution of states under dissipation. For example if we realize the Lindblad operator

$$
L=\left(\begin{array}{cccc}
-0.29878 & 0.32041 & 0.71143 & 0.29878 \\
-0.93333 & 0.36065 & -1.11807 & 0.93333 \\
0.52544 & 0.28336 & -1.53610 & -0.52544 \\
0.48211 & 0.22501 & 1.97634 & -0.48211
\end{array}\right),
$$

and solve the resulting Lindblad equation

$$
\dot{\rho}=\mathcal{L}(\rho)=\sqrt{\Gamma}\left(2 L \rho L^{+}-L^{+} L \rho-\rho L^{+} L\right),
$$

we obtain the density matrix as shown in Figure 4. We may also obtain the density matrices from the relevant Lindblad operators in this simple case using the Baumgartner/Narnhofer formula

$$
\rho^{*}=\frac{\left(L^{+} L\right)^{-1}}{\operatorname{Tr}\left[\left(L^{+} L\right)^{-1}\right]} .
$$

The result is the Bell state $\left|\phi^{+}\right\rangle$. Of course, we may also solve for any other Liouville operator and obtain the corresponding quantum mechanical state.

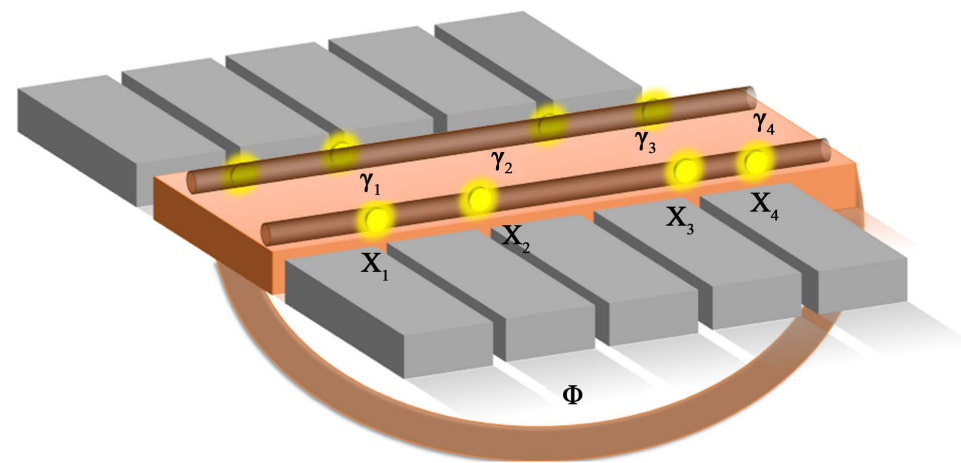

Figure 3. Two Majorana QuBits are encoded in 8 Majorana fermions in two strongly spin-orbit coupled quantum wires (yellow spots). The superconducting substrate can be tunnel-coupled to Josephson-junction with a well-defined phase difference $\Phi$. The position of the Majorana fermions can be tuned by additional side-gates (in grey). 


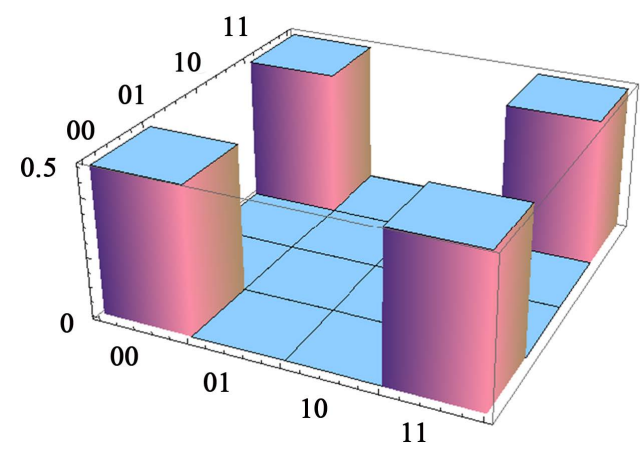

Figure 4. Density matrix $\rho$ resulting from the solution for Equation (11). The entries label the different components of the density matrix. The result corresponds exactly to the Bell state $\left|\phi^{+}\right\rangle$.

In a similar way we can also obtain the necessary certain Lindblad operator $L$ as in Equation (10) for a predefined density matrix $\rho$ in Equation (11). Indeed, this was the process to arrive at Equation (10). Both cases represent elementary numerical calculations.

The corresponding tunneling amplitudes are given by

$$
t_{x, n, m}=\left(L_{x, n, m}[1,2]\right)^{2},
$$

and since the tunnel amplitudes should be tunable by the distance $x$ of the MBSs or side gates as in Figure 3 we may also obtain the necessary configuration. Mind that negative $t_{x, n, m}$ may occur due to the fact that we need to take manifold tunnel processes into account and quantum mechanical phases between the different tunnel processes cannot be simply gauged away, similar to Aharanov-Bohm processes [19].

The process of obtaining the Lindblad operator for a specific state, calculating the necessary tunnel amplitudes and then changing the experimental parameters as tunnel amplitudes and distances on the sample in such a way as to realize the necessary tunnel amplitudes corresponds to the process of tuning the dissipation.

However, in experiment the tunability of $t_{x, n, m}$ will be limited. Nonetheless, this scheme provides a way for good initialization of the Majorana fermion array as in Figure 2 since by coupling them to local phonons we will produce an entangled state which is well defined and can be taken as the starting point e.g. for braiding operations leading to different entangled states. Nonetheless, including the corresponding charge read out operations we also have a universal quantum computer.

\section{Conclusion}

We have considered two examples for dissipative evolutions of the state of Majorana fermions. We have shown that certain states are eigenstates of the dissipative process and therefore dissipation provides a possibility for quantum computation. While the first proposal involving only a simple tunneling term to a metallic lead has been shown to be insufficient for the creation of interesting states, coupling the Majorana fermions to local phonons provided a possibility for universal quantum computation in combination with additional two-QuBit operations. Even if the tunability of the couplings to the local phonons is limited, this scheme still provides a way for well-defined initialization of single Majorana QuBits in an entangled quantum state.

\section{Acknowledgements}

The author would like to David Reeb for many helpful discussions and clarifications during this work.

\section{References}

[1] Alicea, J., Oreg, Y., Refael, G., von Oppen, F. and Fisher, M.P.A. (2011) Non-Abelian Statistics and Topological Quantum Information Processing in 1D Wire Networks. Nature Physics, 7, 412. http://dx.doi.org/10.1038/nphys1915

[2] Kitaev, A.Y. (2001) Unpaired Majorana Fermions in Quantum Wires. Physics-Uspekhi, 44, 131. http://dx.doi.org/10.1070/1063-7869/44/10S/S29 
[3] Oreg, Y., Refael, G. and von Oppen, F. (2010) Helical Liquids and Majorana Bound States in Quantum Wires. Physical Review Letters, 105, Article ID: 177002. http://dx.doi.org/10.1103/PhysRevLett.105.177002

[4] van Heck, B., Akhmerov, A.R., Hassler, F., Burrello, M. and Beenakker, C.W.J. (2012) Coulomb-Assisted Braiding of Majorana Fermions in a Josephson Junction Array. New Journal of Physics, 14, Article ID: 035019. http://dx.doi.org/10.1088/1367-2630/14/3/035019

[5] Mourik, V., Zuo, K., Frolov, S.M., Plissard, S.R., Bakkers, E.P.A.M. and Kouwenhoven, L.P. (2012) Signatures of Majorana Fermions in Hybrid Superconductor-Semiconductor Nanowire Devices. Science, 336, 1003. http://dx.doi.org/10.1126/science.1222360

[6] Stern, A. (2010) Non-Abelian States of Matter. Nature, 464, 187-193. http://dx.doi.org/10.1038/nature08915

[7] Beenakker, C.W.J. (2013) Search for Majorana Fermions in Superconductors. Annual Review of Condensed Matter Physics, 4, 113. http://dx.doi.org/10.1146/annurev-conmatphys-030212-184337

[8] Budich, J.C., Walter, S. and Trauzettel, B. (2012) Failure of Protection of Majorana Based Qubits against Decoherence. Physical Review B, 85, Article ID: 121405. http://dx.doi.org/10.1103/PhysRevB.85.121405

[9] Diehl, S., Micheli, A., Kantian, A., Kraus, B., Büchler, H.P. and Zoller, P. (2008) Quantum States and Phases in Driven Open Quantum Systems with Cold Atoms. Nature Physics, 4, 878-883. http://dx.doi.org/10.1038/nphys1073

[10] Kliesch, M., Barthel, T., Gogolin, C., Kastoryano, M. and Eisert, J. (2011) Dissipative Quantum Church-Turing Theorem. Physical Review Letters, 107, Article ID: 120501. http://dx.doi.org/10.1103/PhysRevLett.107.120501

[11] Verstraete, F., Wolf, M.M. and Ignacio Cirac, J. (2009) Quantum Computation and Quantum-State Engineering Driven by Dissipation. Nature Physics, 5, 633. http://dx.doi.org/10.1038/nphys1342

[12] Groth, C.W., Michaelis, B. and Beenakker, C.W.J. (2006) Counting Statistics of Coherent Population Trapping in Quantum Dots. Physical Review B, 74, Article ID: 125315. http://dx.doi.org/10.1103/PhysRevB.74.125315

[13] Uranga-Pina, L. and Tremblay, J.C. (2014) Relaxation Dynamics in Quantum Dissipative Systems: The Microscopic Effect of Intramolecular Vibrational Energy Redistribution. Journal of Chemical Physics, 141, Article ID: 074703. http://dx.doi.org/10.1063/1.4892376

[14] Walter, S., Schmidt, T.L., Børkje, K. and Trauzettel, B. (2011) Detecting Majorana Bound States by Nanomechanics. Physical Review B, 84, Article ID: 224510. http://dx.doi.org/10.1103/PhysRevB.84.224510

[15] Weiss, U. (2012) Quantum Dissipative Systems. World Scientific, London.

[16] Nayak, C., Simon, S.H., Stern, A., Freedman, M. and Sarma, S.D. (2008) Non-Abelian Anyons and Topological Quantum Computation. Reviews of Modern Physics, 80, 1083-1159. http://dx.doi.org/10.1103/RevModPhys.80.1083

[17] Freedman, M., Nayak, C. and Walker, K. (2006) Towards Universal Topological Quantum Computation in the v=5/2 Fractional Quantum Hall State. Physical Review B, 73, Article ID: 245307. http://dx.doi.org/10.1103/PhysRevB.73.245307

[18] Hassler, F., Akhmerov, A.R., Hou, C.-Y. and Beenakker, C.W.J. (2010) Anyonic Interferometry without Anyons: How a Flux Qubit Can Read out a Topological Qubit. New Journal of Physics, 12, Article ID: 125002. http://dx.doi.org/10.1088/1367-2630/12/12/125002

[19] Maier, S., Dahlhaus, J.P. and Komnik, A. (2010) Spin-Polarized Current Generation and Detection by a Double Quantum Dot Structure. Physical Review B, 81, Article ID: 075110. http://dx.doi.org/10.1103/PhysRevB.81.075110 